\title{
Boundary-field-driven control of discontinuous phase transitions on hyperbolic lattices
}

\author{
Yoju Lee ${ }^{1}$, Frank Verstraete ${ }^{1,2}$, and Andrej Gendiar ${ }^{3}$ F $^{\text {F }}$ \\ 1 Faculty of Physics, University of Vienna, Boltzmanngasse 5, A-1090 Vienna, Austria \\ ${ }^{2}$ Ghent University, Department of Physics and Astronomy, Krijgslaan 281-S9, B-9000 Gent, Belgium and \\ 3 Institute of Physics, Slovak Academy of Sciences, SK-845 11, Bratislava, Slovakia
}

(Dated: August 25, 2016)

\begin{abstract}
The multistate Potts models on two-dimensional hyperbolic lattices are studied with respect to various boundary effects. The free energy is numerically calculated using the Corner Transfer Matrix Renormalization Group method. We analyze phase transitions of the Potts models in the thermodynamic limit with respect to contracted boundary layers. A false phase transition is present even if a couple of the boundary layers are contracted. Its significance weakens, as the number of the contracted boundary layers increases, until the correct phase transition (deep inside the bulk) prevails over the false one. For this purpose we derive a thermodynamic quantity, the so-called bulk excess free energy, which depends on the contracted boundary layers and memorizes additional boundary effects. In particular, the magnetic field is imposed on the outermost boundary layer. While the boundary magnetic field does not affect the second-order phase transition in the bulk if suppressing all the boundary effects on the hyperbolic lattices, the first-order (discontinuous) phase transition is significantly sensitive to the boundary magnetic field. Contrary to the phase transition on the Euclidean lattices, the discontinuous phase transition on the hyperbolic lattices can be continuously controlled (within a certain temperature coexistence region) by varying the boundary magnetic field.
\end{abstract}

PACS numbers: 05.50.+q, 05.70.Jk, 75.10.Hk, 75.40.Mg

\section{INTRODUCTION}

Negatively curved surfaces have been studied experimentally including lattice dislocations of solid-state crystals with non-Euclidean properties, e.g., in various magnetic nanostructures ${ }^{1 / 3}$, or in materials showing a conical geometry ${ }^{4}$. On the other hand, theoretical interest has been focused on the geometry of anti de Sitter (AdS) space ${ }^{516}$ and the complex (e.g. neural) networks $s^{718}$. The nontrivial boundary structure of finite hyperbolic spaces plays an essential role in the analysis of AdS space by means of entanglement entropy 9 -11 The mutual relations among condensed-matter physics, the general theory of relativity, and the conformal field theory (CFT) enrich the interdisciplinary research, such as AdS-CFT correspondence $\mathrm{e}^{12}$.

In 2007, we proposed a way to generalize the Corner Transfer Matrix Renormalization Group (CTMRG) algorithm to a simple hyperbolic surface $\frac{13[14}{\text {. The current }}$ study extends our recent generalization to arbitrary regular hyperbolic geometry ${ }^{15}$, and we focus on the complexity of the boundary effects of hyperbolic lattice geometry. Because no satisfactory analytic studies are available yet, we analyze multistate spin models on hyperbolic lattices numerically. In particular, we focus on phase transitions, which require special treatment because of the non-negligible boundary effect, as they have been suppressed thus far. The CTMRG algorithm enables us to calculate the free energy accurately, including all thermodynamic functions in thermal equilibrium. The free energy approach of the current work has been chosen because it incorporates all the non-negligible boundary effects of the models on hyperbolic lattices. The current study is intended to provide complementary and miss- ing information with respect to the boundary effects performed by Monte Carlo (MC) simulations ${ }^{16}$ 19].

Hyperbolic lattice geometry is known for exhibiting strong boundary effects, which prevent the MC simulations from performing sufficiently precise calculations to classify the phase transitions by means of the free energy. The reason lies in the large fluctuations coming from the rich boundary structure: the boundary size grows exponentially, as the diameter (the shortest distance from the lattice center to the boundary) increases linearly. Hasegawa et al. studied the Ising model via MC simulations, and they determined the phase transition by gradually contracting a couple of the boundary layers ${ }^{20}$. The aim of this paper is to perform a thorough numerical analysis of the multistate spin models by CTMRG with respect to the boundary layers, which are gradually contracted from the entire lattice in the thermodynamic limit.

The paper is organized as follows: In Sec. II we briefly classify an infinite set of hyperbolic lattices by a pair of two integers $\{p, q\}$. We consider the multistate Potts model on the negatively curved lattice surfaces. Setion. III A is devoted to the numerical CTMRG analysis of the first- and second-order phase transitions on square and Bethe lattices. These two lattices are chosen because they are exactly solvable and can serve as the benchmarks for the future studies. This Section can be skipped if the reader is familiar with numerical analysis of phase transitions using thermodynamic functions. Section III B contains the core of this work, where the dependence of the free energy on the gradually contracted layers is shown. We generalize our study to classify $Q$-state Potts models on regular hyperbolic lattice geometries. A concise study of the phase transition deeply inside the infinite bulk is 
given when contracting the infinite number of boundary layers. A phase-coexistence region is specified as a temperature interval, within which the first-order phase transition can be controlled by varying the magnetic field on the boundary layer. Section IV is devoted to discussions and concluding remarks.

\section{LATTICE GEOMETRY AND SPIN MODEL}

Consider a regular two-dimensional lattice (a curved surface) formed by the tessellation of congruent polygons with $p$ sides (referred to as the $p$-gons), and the coordination number $q$ is fixed for the entire lattice. Such a regular lattice can be described by a pair of integers, $\{p, q\}$, known as the Schläfli symbo ${ }^{21}$. Depending on the choice of the two integers $p \geq 3$ and $q \geq 3$, the Schläfli symbol can describe either Euclidean (flat) or curved (spherical or hyperbolic) lattice geometries. If the condition $(p-2)(q-2)=4$ holds, only the following three regular Euclidean two-dimensional lattices exist: the triangular $\{3,6\}$ lattice, the square $\{4,4\}$ lattice, and the honeycomb $\{6,3\}$ lattice. The inequality $(p-2)(q-2)<4$ describes five spherically curved lattices of finite size. However, if $(p-2)(q-2)>4$, an infinite set of hyperbolic lattice geometries can be constructed by tessellation. Each hyperbolic lattice has a particular constant and negative Gaussian curvature, provided that the size of any $p$-gon side is identical21.

Although an arbitrary hyperbolic lattice $\{p, q\}$ of infinite size forms a negatively curved surface, none of the lattices can be embedded in the three- or finitedimensional space. Hence, the Hausdorff dimension is infinite for an arbitrary hyperbolic lattice $\{p, q\}$, provided that the thermodynamic limit is taken. To visualize the hyperbolic lattice $\{p, q\}$, it is useful to project each lattice onto a unitary circle; this mapping is known as the Poincaré disk representation 22 . In such a representation, the congruent $p$-gons (all of the polygons have an identical size and shape on the entire lattice) are deformed if displayed graphically on the disk. The size of the $p$ gons decreases toward the circumference of the disk; the circumference represents the lattice boundary (in the infinity).

At first we specify the size of the lattice in terms of the radius $L$, which is well-visible if the hyperbolic lattice is graphically represented in the Poincaré disk. Let the integer $L$ denotes the actual radius of the lattice beginning in a central lattice site. An example of a hyperbolic lattice geometry $\{5,4\}$ is shown in Fig. 1 (on the left), where the smallest lattice radius with $L=1$ contains four regular pentagons. If $L=2$, the total number of the pentagons is $4+20$, etc. To enhance the $L$-dependence, i.e., the layer structure, the inner layers of the lattice are depicted by the distinct intensity of the gray color. Now it is obvious how the total number of the $p$-gons grows exponentially with respect to the linear increase of the radius $L$ (generalization to any hyperbolic lattice $\{p, q\}$
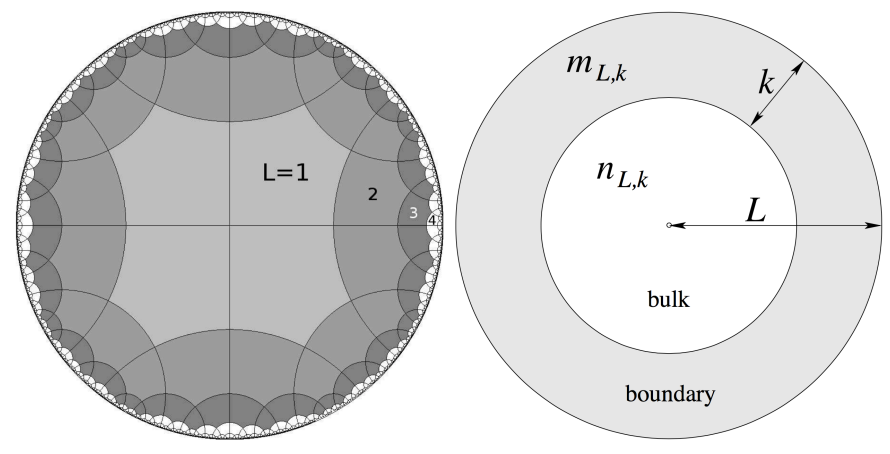

FIG. 1: The schematic picture of the hyperbolic lattice $\{5,4\}$ in the Poincaré disk representation. The entire hyperbolic lattice is composed of $L$ concentric layers, where $L$ is referred to as the lattice size (the radius), which enumerates the layers from the center outward. The inner sublattice, the bulk, has $n_{L, k}$ sites with the diameter $L-k$ of the layers. The outer layers, the boundary, are enumerated by $k$ and consists of $m_{L, k}$ sites.

is straightforward).

\section{A. Boundary layers}

To characterize the boundary layers as a hyperbolic lattice, we ascribe the boundary layers to an integer variable $k$, which enumerates the number of the outermost layers, $k=1,2,3, \ldots$ (provided that $L>k$ ). Whenever we refer to the bulk properties of the (hyperbolic) lattice, one should consider such an innermost sublattice, which has the radius $L-k$ infinite; or, numerically, $L-k$ is sufficiently large so that all thermodynamic functions (normalized to the lattice site) has completely converged. Let $N$ be the total number of the lattice vertices (the sites) for a given diameter $L$. If $k>0$, the entire lattice can be thought of as a system composed of two parts: the bulk subsystem containing $L-k$ inner layers and the $k$ outer boundary layers. The total number of the sites is an integer function of $L$ and is denoted by $\mathcal{N}_{\{p, q\}}^{(L)}$, which depends on the lattice geometry. The inner $L-k$ layers and the outer $k$ layers, respectively, consist of $n_{L, k}$ and $m_{L, k}$ sites, i.e., $\mathcal{N}_{\{p, q\}}^{(L)}=n_{L, k}+m_{L, k}$, as schematically sketched in Fig. 1 (on the right).

If we evaluate the ratio between the boundary sites $m_{L, k}$ and the inner bulk sites $n_{L, k}$, we aim to quantify the significance of the boundary sites, particularly,

$$
R_{k}=\lim _{L \rightarrow \infty} \frac{m_{L, k}}{n_{L, k}} .
$$

Recall that $R_{1 \leq k<+\infty}=0$ for the Euclidean lattice geometries. However, the ratio $R_{k}$ is always positive for the hyperbolic lattices, as shown in Fig. 2. It is instructive to stress that $R_{k=1}>2$ for the three selected lattices, which means that the number of the boundary sites is at least twice as large as the inner $L-1$ sites, even in 


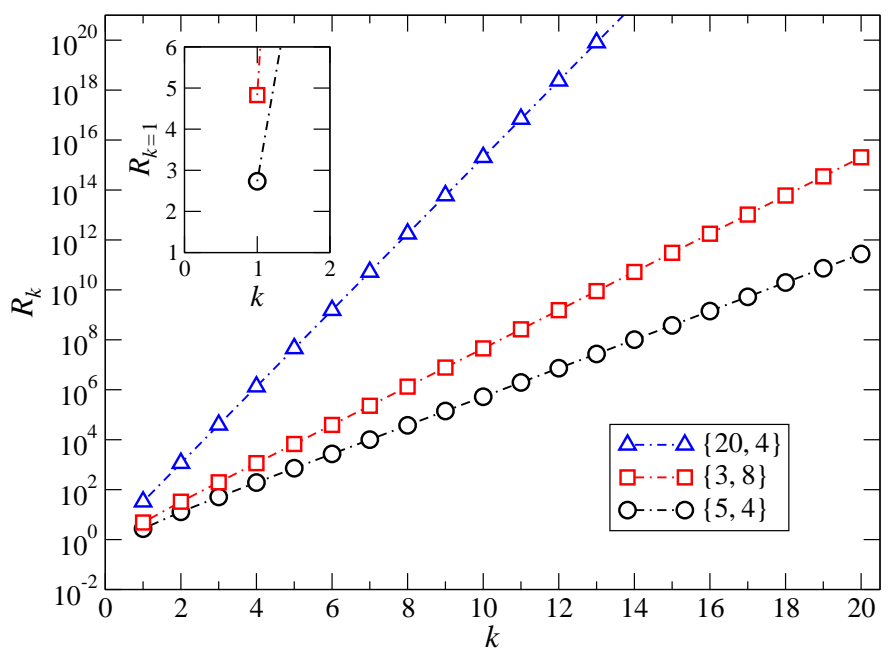

FIG. 2: (Color online) The scaling ratio $R_{k}$ between the boundary and the bulk layers is calculated for the three selected hyperbolic lattices $\{20,4\},\{3,8\}$, and $\{5,4\}$ in the thermodynamic limit $(L \rightarrow \infty)$. The inset shows the detail for the outermost layer when $k=1$.

the thermodynamic limit $L \rightarrow \infty$ (the inset). Or, to be more specific, $R_{1}=2.732,4.824$, and 32.971 on the lattices $\{5,4\},\{3,8\}$, and $\{20,4\}$, respectively. This clearly implies that an enormous number of sites lies on the outermost boundary layer, which significantly influence the inner (bulk) properties that we study in this paper.

\section{B. Potts Model}

Having defined the lattice geometry $\{p, q\}$, now we consider a relevant multistate spin system on those underlying lattices. Let each lattice vertex (site) be a multistate spin variable interacting with the $q$ nearest neighboring spins in each $p$-gon. We study the $Q$-state Potts mode 23 with the nearest-neighbor coupling $J$ (preferably the ferromagnetic one, $J>0$, to avoid frustration for odd $p$ ), whose Hamiltonian has the form

$$
\mathcal{H}=-J \sum_{\langle\langle i, j\rangle\rangle} \delta_{s_{i}, s_{j}}-h \sum_{\langle\langle i\rangle\rangle} \delta_{s_{i}, 0}-h_{b} \sum_{\langle i\rangle_{\mathrm{b}}} \delta_{s_{i}, 0} .
$$

The Kronecker delta $\delta_{s_{i}, s_{j}}$ acts on the two adjacent (nearest-neighbor) $Q$-state spin variables $s_{i}$ and $s_{j}$ positioned on the vertices $i$ and $j$. A uniform constant magnetic field $h$ acting on every spin variable $s_{i}$ is imposed, whereas an independent magnetic field $h_{b}$ is applied on the boundary spins only. Each $Q$-state spin variable $s_{i}$ possesses $Q$ degrees of freedom, $s_{i}=0,1, \ldots, Q-1$, where $Q \geq 2$. The summation $\langle\langle i, j\rangle\rangle$ runs over all the pairs of the nearest-neighboring spins on the entire lattice in the first term. The summation in the second term, $\langle\langle i\rangle\rangle$, specifies the interaction of the magnetic field $h$ with the spin site on the lattice in a preferred spin level $\ell$ (we selected the zeroth spin level, i.e., $\left.\delta_{s_{i}, \ell=0}\right)$. The third term $\langle i\rangle_{\mathrm{b}}$ denotes the interaction of the magnetic field $h_{b}$ with the $m_{L, k=1}$ spin sites located on the outermost boundary layer. When the number of the spin degrees of freedom is set to be as small as $Q=2$, the model is equivalent to the Ising model.

For the calculation of the thermodynamic functions, we apply the generalized CTMRG algorithm ${ }^{15}$, which has been proven to be a powerful numerical method for classical multistate spin lattice models 14 . The CTMRG is a classical counterpart of the Density Matrix Renormalization Group 2425. It has been successfully applied to various spin models on Euclidean and the hyperbolic lattices $13|15| 26|28| 31$. The algorithm is an efficient and accurate tool to calculate the partition function $\mathcal{Z}=\operatorname{Tr} \exp \left(-\mathcal{H} / k_{\mathrm{B}} T\right)$, or eventually, the free energy $\mathcal{F}=-k_{\mathrm{B}} T \ln \mathcal{Z}$, and the related thermodynamic functions. Here, $k_{\mathrm{B}}$ and $T$, respectively, are the Boltzmann constant and temperature. In the following we use the dimensionless units, and we consider $k_{\mathrm{B}}=1$. More specific details of the CTMRG algorithm modified for the hyperbolic lattices are summarized in Refs. 1315.

To summarize the CTMRG idea in brief, the partition function is considered in terms of the tensor product of local Boltzmann weights $\mathcal{W}=\exp \left(-\mathcal{H}_{\{p, q\}} / k_{\mathrm{B}} T\right)$ of the $p$-gonal shape, where the Hamiltonian $\mathcal{H}_{\{p, q\}}$ is a cyclic spin chain of interacting spins within the $p$-gon for a given $\{p, q\}$ lattice geometry. The algorithm is designed to cover the entire lattice with the identical Boltzmann weights so as to satisfy the constant coordination number $q$. The lattice expands iteratively in the algorithm, starting from the $q$ Boltzmann weight sharing the central site. The lattice grows linearly with the diameter $L$ within the renormalization group procedure until the renormalized corner transfer tensors converge. The full convergence is associated with the thermodynamic limit, which is a necessary condition to analyze the phase transitions.

The $Q$-state Potts model is exactly solvable $e^{23}$ on the square lattice $\{4,4\}$, resulting in the phase transition temperature $T_{\mathrm{pt}}=1 / \ln (1+\sqrt{Q})$ for any $Q \geq 2$. In a special case, the two-state Potts model is equivalent to the Ising model, which has an exact solution on the Bethe lattice 5 for arbitrary coordination number $q \geq 3$, and the phase-transition temperature is $T_{\mathrm{pt}}=1 / \ln [q /(q-2)]$. In the following, we refer to these exact phase transition temperatures, which we use for comparison with the numerical results. As we have shown earlier ${ }^{15 \mid 26}$, the thermodynamic properties of the spin models in the bulk on the hyperbolic lattices $\{p \gtrsim 15, q\}$ are, in fact, numerically indistinguishable from those on the Bethe $\{\infty, q\}$ lattices $^{21}$, regardless of the coordination number $q$; cf. Ref. 26. Therefore, we use the hyperbolic lattice $\{20, q\}$ as the Bethe lattice $\{\infty, q\}$. Figure 3 shows the lattice structure in the Poincaré representation of the hyperbolic lattice $\{20,4\}$ (left) being numerically equivalent to the Bethe lattice $\{\infty, 4\}$ (right). The CTMRG algorithm applied to an arbitrary lattice $\{p, q\}$ is designed to join $q$ corner transfer tensors around a central site $\sigma_{c}$; for better visibility, Fig. 3does not display the spin $\sigma_{c}$ at the center 


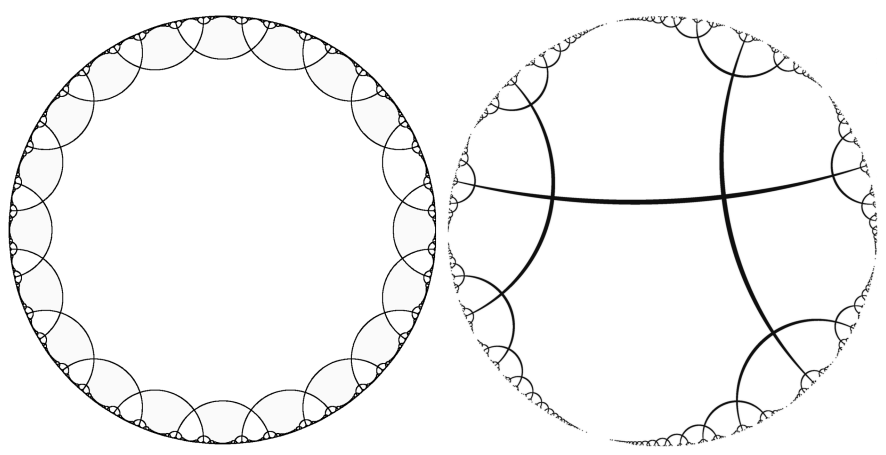

FIG. 3: The hyperbolic lattice geometries $\{20,4\}$ (left) and $\{\infty, 4\}$ (right) are numerically indistinguishable if the bulk properties of the spin models are considered.

of the unitary Poincaré circle for the two lattices ${ }^{15}$. Having compared the relative error $\varepsilon$ at the phase-transition temperature by the CTMRG algorithm with respect to the analytic result, we obtained high accuracy, $\varepsilon \lesssim 10^{-5}$ for $Q=2$ and $\{20, q\}$.

\section{RESULTS}

There are two independent calculations in CTMRG to specify the phase transitions.

(i) The simplest one is to calculate the expectation value for the spin (the spontaneous magnetization)

$$
M_{\{p, q\}}=\frac{Q\left[\operatorname{Tr}\left(\delta_{\sigma_{c}, 0} \rho\right)\right]-1}{Q-1}
$$

where $\sigma_{c}$ is the $Q$-state spin variable located in the central site of the lattice and $\rho$ is a reduced density matrix that is the partial configuration sum of the product of $q$ corner transfer tensors 15 . This approach leads to an accurate determination of the phase transition temperature in the bulk ${ }^{13 / 26}$. The effects of the lattice boundaries are strongly suppressed in this type of calculation. Therefore, the spontaneous magnetization in Eq. (2) has been used as a reliable criterion to determine the phase transition in the bulk. Analogously, the nearest-neighbor (NN) correlation energy,

$$
E_{\{p, q\}}=-\operatorname{Tr}\left(J \delta_{\sigma_{c}, \sigma_{c+1}} \rho\right)
$$

can also be evaluated between two neighboring spins in the center of the lattice. Here, the phase transition can be extracted from the singularity (a diverging peak) of the specific heat, which is proportional to taking the numerical derivative of $E_{\{p, q\}}$ with respect to temperature.

(ii) The second, and independent way to find the phase transition is to evaluate the free energy of the entire system, and the boundary effects contribute significantly to the free energy. We have recently derived recursive expressions for the free energy per spin site, $\mathcal{F}_{\{p, q\}}$ for any multistate spin system including the boundary magnetic field $h_{b}$. A detailed analysis of the free energy per site is given in Ref. 15. Thus, the free energy per spin site (expressed in the thermodynamic limit) has the form

$$
\mathcal{F}_{\{p, q\}}=-k_{\mathrm{B}} T \lim _{L \rightarrow \infty} \frac{\ln \mathcal{Z}_{\{p, q\}}^{(L)}}{\mathcal{N}_{\{p, q\}}^{(L)}}
$$

We can directly use Eq. 4 since the partition function $\mathcal{Z}$ can be calculated to a high numerical accuracy by the CTMRG algorithm, and the total number of the spin sites $\mathcal{N}_{\{p, q\}}^{(L)}=n_{L, 0}+m_{L, 0}$ has an analytic expression for any $\{p, q\}$ geometry 15 . Recall, that the numerical derivatives of the free energy with respect to temperature $T$ or the magnetic field $h$, yield the correct thermodynamic functions with the singular behavior at the phase transition. Below, we refer to the following thermodynamic functions: the normalized entropy per spin,

$$
S_{\{p, q\}}=-\frac{\partial \mathcal{F}_{\{p, q\}}}{\ln (Q) \partial T},
$$

the internal energy,

$$
U_{\{p, q\}}=-T^{2} \frac{\partial\left(\mathcal{F}_{\{p, q\}} / T\right)}{\partial T},
$$

and the specific heat,

$$
C_{\{p, q\}}=\frac{\partial_{T} U_{\{p, q\}}}{\partial T} .
$$

The normalization factor $\ln (Q)$ in Eq. (5) is chosen to restrict the entropy to the interval $0 \leq S_{\{p, q\}} \leq 1$ (note that $S_{\{p, q\}}=0$ at $T=0$ and $S_{\{p, q\}}=1$ at $T \rightarrow \infty$ in this work).

\section{A. Continuous versus discontinuous phase transitions}

For tutorial purposes, we select the 2-state Potts model to represent a second-order (continuous) phase transition, and the 5-state Potts model, which is known for exhibiting a first-order (discontinuous) phase transition on the square lattice $\{4,4\}$. The $Q$-state Potts models results in the second-order phase transitions if $2 \leq Q \leq 4$, whereas the first-order phase transitions arise if $Q \geq 5$ on the two-dimensional Euclidean lattices 23 . We first concentrate on the simplest case: no boundary layers are contracted $(k=0)$ and no magnetic field is imposed on the boundary layer $\left(h_{b}=0\right)$, whereas the thermodynamic limit is taken $(L \rightarrow \infty)$.

\section{Ising model on the square and Bethe lattices}

First, we investigate the exactly solvable Ising model on the square lattice $\{p=4,4\}$ and the Bethe $\{p \rightarrow \infty, 4\}$ 

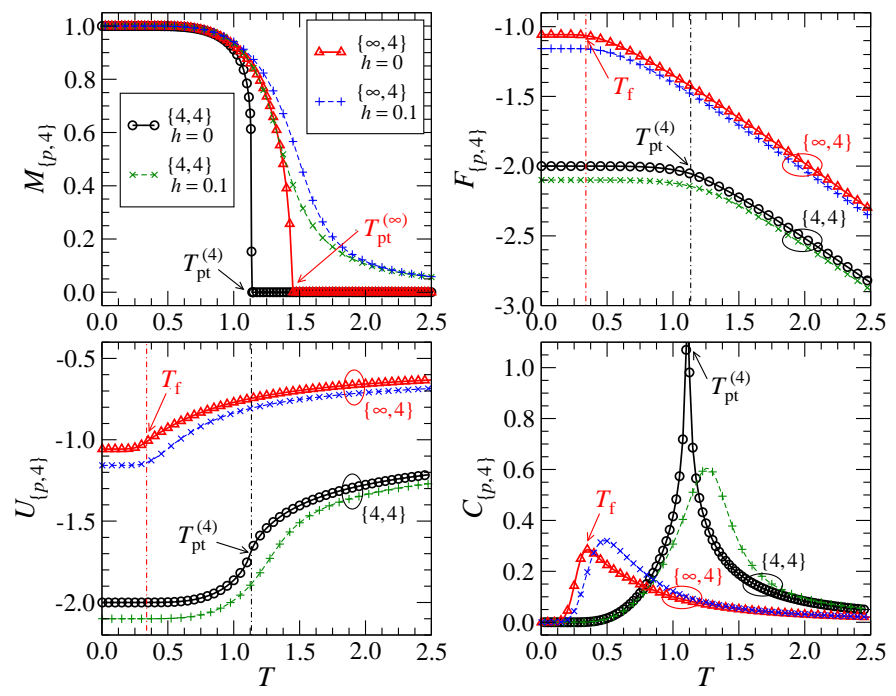

FIG. 4: (Color online) The Ising model: temperature dependence of the spontaneous magnetization $M_{\{p, 4\}}$, the free energy per site $\mathcal{F}_{\{p, 4\}}$, the internal energy $U_{\{p, 4\}}$, and the specific heat $C_{\{p, 4\}}$ calculated on the square lattice $\{4,4\}$ (black circles) and the Bethe lattice $\{\infty, 4\}$ (red triangles). Response of the model to the external magnetic fields $h=0$ (green symbol ' $x$ ') and $h=0.1$ (blue symbol ' + ') is shown. The vertical dot-dashed lines help in identifying the correct phase transition temperature $T_{\mathrm{pt}}^{(p)}$ including the false one located at $T_{\mathrm{f}} \approx 0.339$.

lattice, and we compare the correctness of the phasetransition temperature with the exact one. The Ising model exhibits an order-disorder phase transition at critical temperature $T_{\mathrm{pt}}^{(p=4)}=1 / \ln (1+\sqrt{2}) \approx 1.13459 \ldots$ on the square lattice and $T_{\mathrm{pt}}^{(p \rightarrow \infty)}=1 / \ln 2 \approx 1.44269 \ldots$ on the Bethe lattice. The four typical thermodynamic functions with respect to temperature for the Ising model are shown in Fig. 4. The phase transition temperature $T_{\mathrm{pt}}^{(p)}$ is evident from the typical behavior at zero magnetic field $h$. The dependence of the four functions at the nonzero field, $h=0.1$, is also included. The secondorder phase transition temperature is well-visible as the diverging peak in the specific heat [cf. Eq. (7)], and the free energy does not exhibit any non-analytic behavior.

Since the spontaneous magnetization of the Ising model is calculated as the expectation value of the spin in the center of the lattice (where all the boundary effects are suppressed), the phase-transition temperature agrees with the analytic result for both the square and the Bethe lattices. It is located at the temperature $T_{\mathrm{pt}}^{(p)}$, which separates the ordered phase with $M_{\{p, 4\}}>0$ from the disordered one, where $M_{\{p, 4\}}=0$ at $h=0$. The calculation of the remaining three thermodynamic functions $\mathcal{F}_{\{p, 4\}}, U_{\{p, 4\}}$, and $C_{\{p, 4\}}$ includes the boundaries. Therefore, the Ising model on the Bethe lattice shows no evident singularity at $T_{\mathrm{pt}}^{(\infty)}$ if the specific heat $C_{\{\infty, 4\}}$ for $h=0$ is calculated (cf. $M_{\{\infty, 4\}}$ ). There is, however, a remarkable (but non-diverging) maximum in $C_{\{\infty, 4\}}$ at
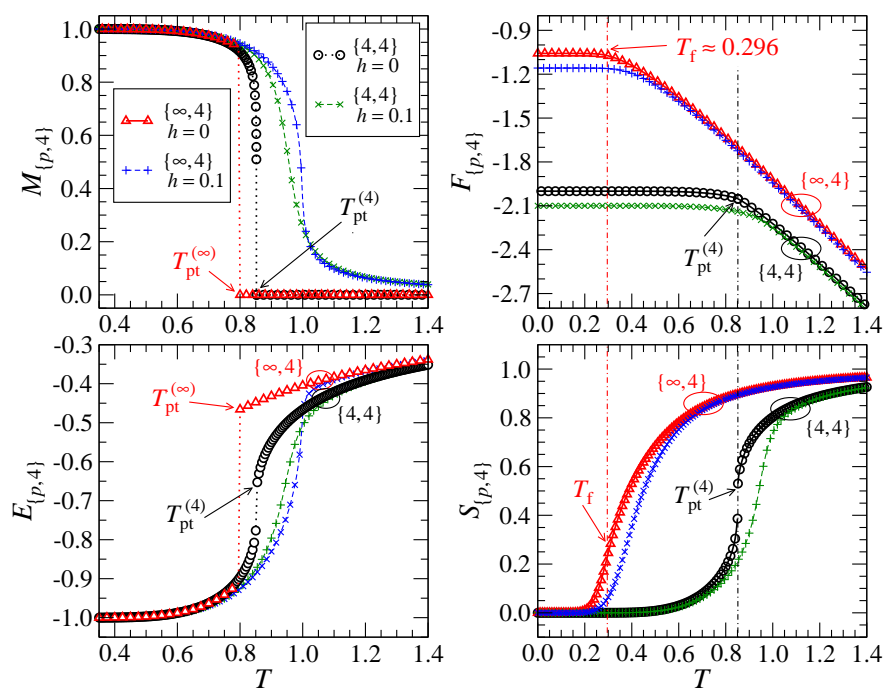

FIG. 5: (Color online) The 5-state Potts model: the spontaneous magnetization $M_{\{p, 4\}}$, the free energy per site $\mathcal{F}_{\{p, 4\}}$, the nearest-neighbor correlation energy $E_{\{p, 4\}}$, and the normalized entropy per site $S_{\{p, 4\}}$ are plotted with respect to temperature on the square and the Bethe lattices for $h=0$ and $h=0.1$. The false phase transition temperature is located at $T_{\mathrm{f}} \approx 0.296$ on the Bethe lattice at $h=0$.

significantly lower temperature, which we do not associate with a phase transition. In the following, we refer to that non-diverging maximum in the specific heat as the false phase transition, which is located at temperature $T_{\mathrm{f}} \approx 0.339$. The non-existence of the phase transition on the entire Bethe lattice is not surprising, as was pointed out in Ref. 27. Our numerical analysis using the free energy, thus, reflects such a feature on the hyperbolic lattice, and it will be resolved below.

\section{5-state Potts model on the square and Bethe lattices}

The first-order phase transition is associated with a sudden (discontinuous) change of some thermodynamic functions at the phase transition. Specifically, a nonzero latent heat $\Delta E\left(T_{\mathrm{pt}}\right)>0$ is associated with the discontinuous phase transition at $T_{\mathrm{pt}}$, in particular, $\Delta E \equiv$ $U\left(T_{\mathrm{pt}}^{+}\right)-U\left(T_{\mathrm{pt}}^{-}\right) \propto E\left(T_{\mathrm{pt}}^{+}\right)-E\left(T_{\mathrm{pt}}^{-}\right)$. The nonzero latent heat is the typical signature of the first-order phase transition, which originates in the nonanalytic behavior of the free energy at the phase transition. There is a typical kink (crossover) in the free energy per site, which can be precisely determined by CTMRG after introducing free and fixed boundary conditions on the Euclidean lattices 32 . To visualize this effect, we plotted a couple of the thermodynamic functions for the 5-state Potts model in Fig. 5. To avoid showing a qualitative similarity of the internal energy $U_{\{p, 4\}}$ and the specific heat $C_{\{p, 4\}}$ (as shown in Fig. 4), we replace them by the NN correlation energy $E_{\{p, 4\}}$ and the normalized entropy $S_{\{p, 4\}}$, respectively. 

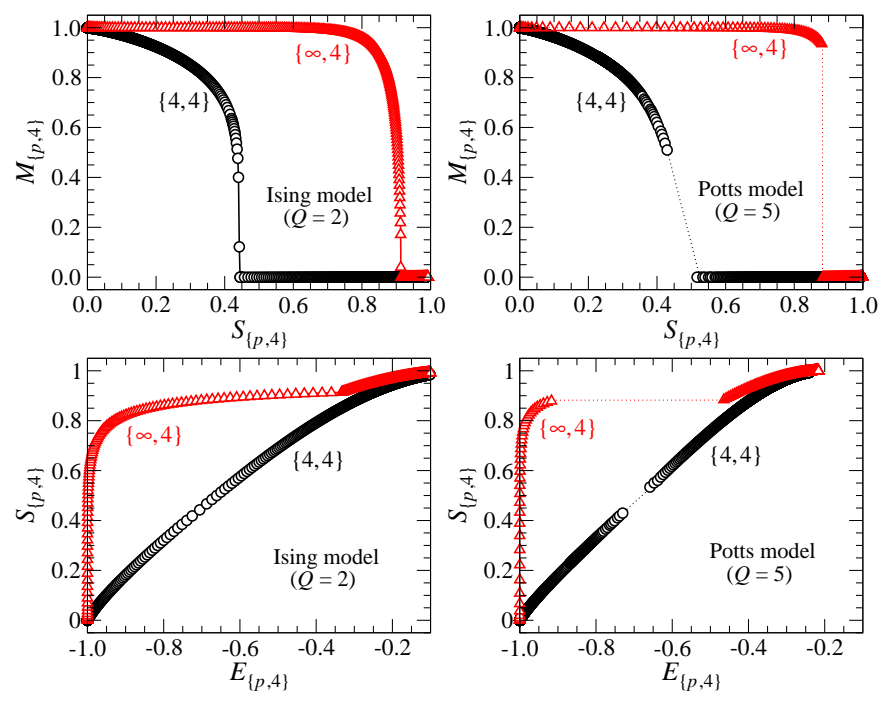

FIG. 6: (Color online) The two top panels show the functional relations between the magnetization $M$ and the entropy $S$. The two bottom panels show the entropy $S$ versus the NN correlation energy $E$. The second-order phase transition is manifested by the continuous behavior of the thermodynamic functions, whereas the first-order phase transition opens the nonzero gaps in the thermodynamic functions.

The evident discontinuity at the phase transition occurs in $M_{\{4,4\}}, E_{\{4,4\}}$, and $S_{\{4,4\}}$ at $h=0$ [including the less visible kink at $T_{\mathrm{pt}}^{(4)}$ in $\mathcal{F}_{\{4,4\}}$, for details, cf. Ref. 32]. Obviously, the nonzero latent heat is present, and the method correctly captures the first-order phase transition temperature, which has an analytic solution 23 $T_{\mathrm{pt}}^{(4)}=1 / \ln (1+\sqrt{5})=0.8515 \ldots$ on $\{4,4\}$.

On the other hand, the 5-state Potts model on the Bethe lattice shows different features. If the boundary effects are neglected, a large discontinuous jump emerges in the magnetization $M_{\{\infty, 4\}}$. There is a jump in the NN correlation energy $E_{\{\infty, 4\}}$, which is proportional to a nonzero latent heat, that is the sign of the first-order phase transition in the bulk). However, when analyzing the free energy $\mathcal{F}_{\{\infty, 4\}}$ and the normalized entropy $S_{\{\infty, 4\}}$ on the entire lattice system with the boundary effects, both the free energy and the entropy, remain continuous (analytic) in the entire temperature interval. Again, we have localized the false phase transition, which is located at $T_{f} \approx 0.296$. The vertical dot-dashed lines in Fig. 5 serve as guides for the eye to denote the correct and the false phase transitions.

\section{Basic specifications of discontinuous phase transitions}

To emphasize the differences in the thermodynamic properties of the Potts models on the square and Bethe lattices, we plot mutual functional relations of the thermodynamic functions in Fig. 6. The graphs show the continuous $(Q=2)$ and the discontinuous $(Q=5)$ phase

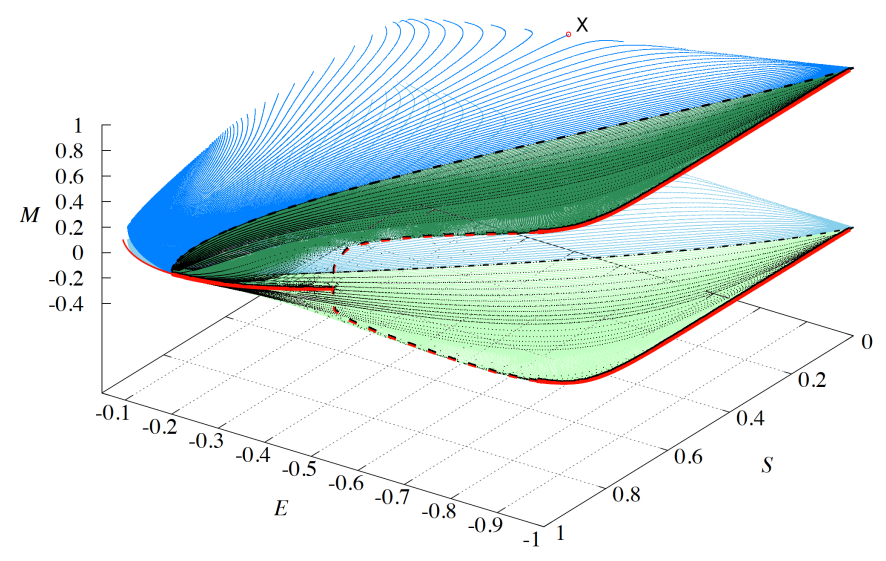

FIG. 7: (Color online) The convex set of $\{E, S, M\}$ for the 5 -state Potts model on the Bethe lattice $\{\infty, 4\}$ forms a twodimensional surface of a shell-like object. Each thick or thin surface curves correspond to the particular flows of $\{E, S, M\}$ with respect to the varying temperature $T$ for given fixed values $J$ and $h$. The data shown are calculated for $h_{b}=k=0$ in the thermodynamic limit $(L \rightarrow \infty)$.

transitions from a different viewpoint. The two top panels depict the functional dependence of the spontaneous magnetization per site $M_{\{p, 4\}}$ on the normalized entropy $S_{\{p, 4\}}$ on the square and the Bethe lattices in the thermodynamic limit. The two bottom graphs show the normalized entropy per site $S_{\{p, 4\}}$ with respect to the NN correlation function $E_{\{p, 4\}}$. All the graphs are evaluated for $J=+1, h=0$, and $h_{b}=0$ within the temperature interval $0.1 \leq T \leq 10$. The Ising model on the both lattices reproduces the second-order phase transition as the continuous functions, whereas the first-order phase transition (ascribed to the case $Q=5$ ) exhibits the discontinuities in the thermodynamic functions.

If one is interested in understanding the global phase structure of various systems, a convex set ${ }^{33134}$ is a useful tool for such a demonstration. The convex set is usually constructed for three typical thermodynamic functions to reveal the details of various types of the phase transitions if plotting their mutual functional dependences. Since the convex sets have been constructed neither for the first-order phase transitions nor for the non-Euclidean lattices, we plot Fig. 7 to display the convex set $\{E, S, M\}$ for the 5-state Potts model on the Bethe lattice. We chose the NN correlation function $E \equiv E_{\{\infty, 4\}}$, the normalized entropy $S \equiv S_{\{\infty, 4\}}$, and the spontaneous magnetization $M \equiv M_{\{\infty, 4\}}$ to construct $\{E, S, M\}$ after having scanned sufficiently wide intervals of the parameters $T, J$, and $h$. In particular, we calculated the thermodynamic functions in the intervals of the temperatures $0.1 \leq T \leq$ 10 , the coupling constants $-2 \leq J \leq+2$, and the uniform magnetic field $-10 \leq h \leq+10$. The constructed convex set has to be understood as the functional set $\{E, S, M\} \equiv\{E(T, J, h), S(T, J, h), M(T, J, h)\}$. The convex set $\{E, S, M\}$ of the 5 -state Potts model forms a two-dimensional, ]semi-open, and curved surface with 
a shell-like structure. The surfaces of the upper and the lower shells, respectively, correspond to $h>0$ and $h<0$. It is worth mentioning that the present convex set differs remarkably from those obtained for the models on the Euclidean surfaces. For instance, if the Ising model on the $\{4,4\}$ lattice is considered (not shown), the convex set covers a smaller surface region and is identical to that evaluated in Ref. 35. The full thick red curves (bordering the front edges) correspond to the case $J=1$ and $h=0$ when the entire temperature interval is scanned. The red dashed curves connecting the full curves serve only as guides for the eyes. The dashed curves denote a discontinuous jump (it is used to connect the two full thick red lines), and the discontinuity cannot by reached by any $T, J$, and $h$. The unique jump is, therefore, explicitly shown in the two panels in Fig. 6 (on the right). The discontinuity can be asymptotically approached for small magnetic fields $h$. The point, at which the full and the two dashed red curves meet is a kind of repeller point at $\{E \approx 0.467, S \approx 0.822, M=0\}$. No discontinuity of that kind has been reported yet. The thin black dashed curves refer to the zero NN spin coupling $(J=0)$; they divide the top (bottom) shell into two regions (the green one with $J>0$ and the blue one with $J<0$ ). The sign of the spontaneous magnetization $M$ coincides with the sign of the magnetic field $h$. Each thin curve on the surface of $\{E, S, M\}$ is parameterized by the temperature $T$ for the fixed values $J$ and $h$. When restricting to the upper shell only, there are three important attractors (fixed points) in the graph: $\mathcal{A}_{\infty}, \mathcal{A}_{1}$, and $\mathcal{A}_{2}$. The attractor $\mathcal{A}_{\infty}$ is connected either with $\mathcal{A}_{1}$ or $\mathcal{A}_{2}$ while varying temperature $0 \leq T<+\infty$. The attractor $\mathcal{A}_{\infty}$ is positioned at $\{E=-0.2, S=1, M=0\}$ at $T \rightarrow \infty$, and it can be reached for arbitrary $J$ and $h$. The two distinct attractors $\mathcal{A}_{1}$ and $\mathcal{A}_{2}$ can be accessed at $T=0$ : the first one at $\{E=-1, S=0, M=+1\}$ and the second one at $\{E=0, S=0, M=0\}$ (not shown due to strong frustration effects caused by the competing antiferromagnetic interaction $J<0$ and the uniform magnetic field, $h \neq 0$; the numerical data are not reliable if the frustration becomes strong, i.e. if $\left.E_{\{p, q\}}>-0.1\right)$. The two attractors $\mathcal{A}_{1}$ and $\mathcal{A}_{2}$ located at $T=0$, which is equivalent to $S=0$ on the graph, are mutually inaccessible by varying the temperature $T$. This is due to a bifurcation point $X$ that separates them (cf. Fig. 7). The bifurcation point is located at $\{E \approx-0.4, S=0, M \approx 0.62\}$ on the upper shell. The top and the bottom shells do not show a mirror symmetry with respect to the plane at $M=0$. The bottom shell surface (for negative $M$ ) is less shallow if compared to the upper one. This is due to the Potts model spin levels, for which the spontaneous magnetization of the 5-state Potts model exhibits five-fold degeneracy of the free energy below the phase transition. The free energy minima at $T<T_{\mathrm{pt}}$ are associated with $Q=5$ projections of the spontaneous magnetization in Eq. (2) being polarized at $T=0$ either to the spin level with the magnetization $M=+1$ or to the four identical levels with $M=-\frac{1}{4}$ (cf. Ref. 32 ), provided that the spon- taneous symmetry-breaking has occurred in the thermodynamic limit [because the magnetic field $h$ is in effect up to the spin level $\ell=0$, the term $h \delta_{\sigma_{i}, \ell=0}$ is used in Hamiltonian (1)]. Apart from the atypical discontinuous region denoted by the thick dashed red curves, there is another interesting feature: both the top and the bottom shells asymptotically reach the zero entropy limit $S \rightarrow 0$ as $T \rightarrow 0$ for the entire spectrum of $E$, which is not accessible on the Euclidean lattice geometry ${ }^{35}$, where the entropy of the bifurcation point is nonzero (i.e., $S>0.5$ for the Ising model on $\{4,4\})$.

\section{B. Analysis of the boundary effects}

As we have mentioned earlier, the boundary of the hyperbolic lattices affect the bulk properties, such as the suppression of the phase transition. At the same time, a false phase transition appears, which has an obvious origin in the rich boundary structure. Therefore, we propose a thermodynamic function for any underlying geometry $\{p, q\}$

$$
\mathcal{B}_{\{p, q\}}^{(k)}=\mathcal{F}_{\{p, q\}}-\mathcal{F}_{\{p, q\}}^{*(k)},
$$

where $\mathcal{F}_{\{p, q\}}$ is the free energy per site [cf. Eq. (4)] of the entire system $L$ in the thermodynamic limit and $\mathcal{F}_{\{p, q\}}^{*(k)}$ is the free energy of the $k$ outermost boundary layers. To avoid confusions, we stress that $\mathcal{B}_{\{p, q\}}^{(k)}$ is not identical to an isolated free energy of the $L-k$ layers. Instead, it is meant to describe a thermodynamic function, that represents bulk excess free energy, $\mathcal{B}_{\{p, q\}}^{(k)}$. It contains all the contributions coming from the $k$ contracted boundary layers (via normalization factors within the bulk, which depends recursively on each of the $k$ boundary factors ${ }^{15}$ ). Such a specific definition of the bulk excess free energy in Eq. (8) has been chosen as the only numerically feasible quantity, that can determine the correct phase transition, including the boundary effects. (For completeness, $\mathcal{B}_{\{p, q\}}^{(k=0)} \equiv \mathcal{F}_{\{p, q\}}$ if $k=0$ only. $)$

\section{Ising model on Bethe lattice for $k \rightarrow \infty$}

First, the bulk excess free energy is tested in two asymptotic regimes: $k=0$ and $k \rightarrow \infty$. We focus on the phase transition analysis in the Ising model $(Q=2)$ on the Bethe lattice $\{\infty, 4\}$. If $k \rightarrow \infty$, we consider the case when both the number of bulk layers, $L-k$, and the number of boundary layers, $k$, are infinite. To achieve this, one can, e.g., take $k=\frac{L}{2}$ and perform the thermodynamic limit $L \rightarrow \infty$. Whenever we refer to the two asymptotic cases $(L \rightarrow \infty$ and $k \rightarrow \infty)$, we mean sufficiently large (finite) values $L$ and $k$, for which any further increase of either of them has not affected the thermodynamic functions in Eqs. (2)-(7) at all. Since these thermodynamic functions are normalized per spin site, they 

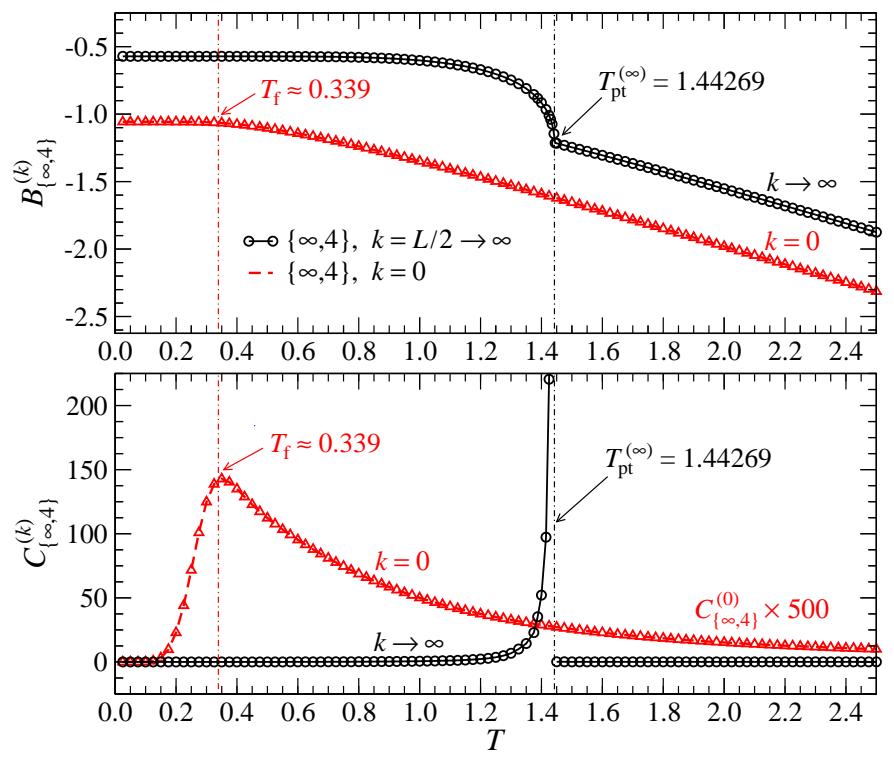

FIG. 8: (Color online) The bulk excess free energy (the upper graph) and the 'specific heat' (the lower graph) with respect to temperature in the thermodynamic limit calculated for the Ising model on the Bethe lattice when $h=0$, regardless of $h_{b}$. The two cases are shown: (1) $k=0, L \rightarrow \infty$ (the circles), i.e. no contracted boundary layers, and (2) $k \rightarrow \infty, L \rightarrow \infty$ (the triangles), i.e. infinitely many boundary layers are contracted while the inner lattice size remains infinite, too. The two dotdashed vertical lines denote the correct and the false phase transition temperatures.

always have to converge completely. Typically, we set $L=6000$ for $k=3000$ (which is the case of $L, k \rightarrow \infty$ ). We have checked that the numerical results remain identical if $k$ is chosen arbitrarily, regardless of the order in which the two limits $k$ and $L$ are taken.

The upper graph of Fig. 8 shows the bulk excess free energy per site, $\mathcal{B}_{\{\infty, 4\}}^{(k)}$, for the Ising model on the Bethe lattice when $k=0$ and $k \rightarrow \infty$. There is an evident singularity in the bulk excess free energy in the correct phase transition, $T_{\mathrm{pt}}^{(\infty)}=1 / \ln 2$. The lower graph shows the second derivative of the bulk excess free energy with respect to temperature,

$$
\mathcal{C}_{\{\infty, 4\}}^{(k)}=-T \frac{\partial^{2} \mathcal{B}_{\{\infty, 4\}}^{(k)}}{\partial T^{2}}
$$

for both $k=0$ and $k \rightarrow \infty$, which we refer to as the "specific heat' in the following (where the term "specific heat" is true only if $k=0$ ). The singularity of the 'specific heat' for $k \rightarrow \infty$ occurs exactly at the phase-transition temperature $T_{\mathrm{pt}}^{(\infty)}=1 / \ln 2$, as is analytically derived in the bulk of the Bethe lattice ${ }^{27}$. The singularity is identical to that in the spontaneous magnetization in Fig. 4. If an arbitrary boundary magnetic field $h_{b}$ is imposed on the boundary layer, both the phase transition temperature $T_{\mathrm{pt}}^{(\infty)}$ and the bulk excess free energy $\mathcal{B}_{\{\infty, 4\}}^{(\infty)}$ remain unaffected by setting any $h_{b} \neq 0$ (not shown). An ad-

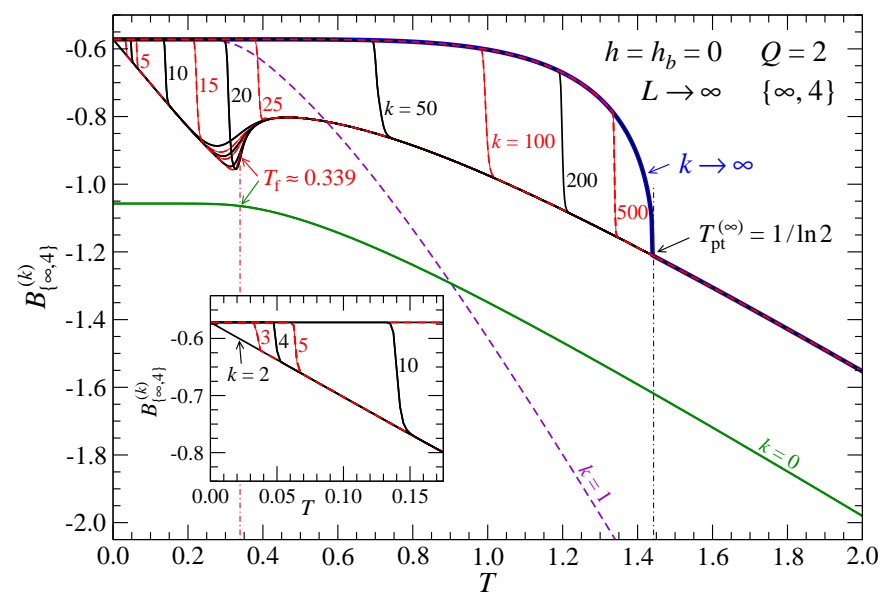

FIG. 9: (Color online) Temperature dependence of the bulk excess free energy per site for the Ising model on the Bethe lattice for a wide range of the contracted boundary layers $k$. Notice the specific region located around the false phase transition $T_{\mathrm{f}}$, where the bulk excess free energy grows, is denoted by the dot-dashed arrows.

ditional remark is inevitable: the square lattice results in $\mathcal{B}_{\{4,4\}}^{(k)} \equiv \mathcal{F}_{\{4,4\}}$, regardless of whether we took $k=0$ or $k \rightarrow \infty$ in the thermodynamic limit. Moreover, if an arbitrary boundary magnetic field $h_{b}$ is imposed, all thermodynamic functions in the bulk are unaffected by it. This is true for any spin model exhibiting a secondorder phase transition on Euclidean lattices, since the boundaries are negligible $\left[R_{k}=0\right.$ (for any $k \geq 0$ )].

Ising model on Bethe lattice for $0 \leq k<\infty$

From the previous analysis we came to understand the importance of contracting an infinite number of the outermost layers in order to detect the correct phase transition in the bulk by means of $\mathcal{B}_{\{\infty, 4\}}^{(\infty)}$. Now, we analyze bulk excess free energy if we gradually contract the individual boundary layers (indexed by $k$ ). The aim is to reveal how the false phase transition at $k=0$ gets suppressed, and the correct phase transition appears at $k \rightarrow \infty$. As an instructive example, the Ising model on the Bethe lattice is again considered. Figure 9 shows the $k$-dependence of the bulk excess free energy with respect to temperature for $h=h_{b}=0$. For comparison, the two limiting cases $k=0$ and $k \rightarrow \infty$ are also plotted. As the number of the contracted boundary layers $k$ increases, the bulk excess free energy for $k=1,2,3, \ldots, \infty$ undergoes a specific regime. We also observe dramatic changes of $\mathcal{B}_{\{\infty, 4\}}^{(k)}$ toward the asymptotics $\mathcal{B}_{\{\infty, 4\}}^{(k \rightarrow \infty)}$ (shown by the thick blue line in the limit $k \rightarrow \infty$ in accord with the upper graph of Fig. 8). We stress an abrupt (continuous) decrease of the bulk excess free energy for $k=3,4, \ldots, 500$. The higher $k$ is, the closer the abrupt decrease in the asymptotic correct phase transition occurs. The false 


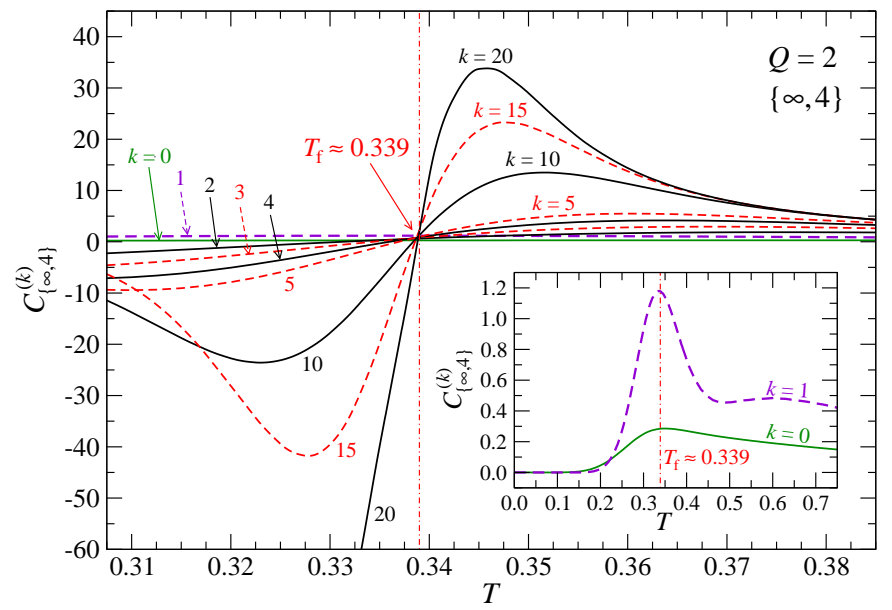

FIG. 10: (Color online) The specific heat vs temperature exhibits the unique behavior around the false phase transition temperature $T_{\mathrm{f}} \approx 0.339$ after the boundary layers $k=0,1,2,3, \ldots, 20$ are gradually contracted. The inset shows the maximum for $k=0$ and $k=1$.

phase transition at $T_{\mathrm{f}} \approx 0.339$ (depicted in Figs. 4 and 8) is denoted by a thin vertical dot-dashed line and is relevant for $0 \leq k \lesssim 20$ in this particular case. It is clear that the bulk excess free energy remains significantly influenced by the lattice boundaries, and the false phase transition $T_{\mathrm{f}}$ prevails over the correct phase transition $T_{\text {pt }}$ for $k \lesssim 20$. If $k=1$, there is a surprisingly distinct asymptotic behavior of the bulk excess free energy $\mathcal{B}_{\{\infty, 4\}}^{(1)}$ at $T>T_{\mathrm{f}}$ compared to $k>1$. This is also manifested in the fact that after contracting the outermost boundary layer, the bulk excess free energy changes dramatically, despite the fact that the majority of lattice sites were disregarded $\left(R_{1}>1\right)$. For illustration purposes, the total number of outermost boundary spins $m_{\infty, k=1}$ is approximately 33 times larger on the lattice geometry $\{20,4\}$ than the spins inside, cf. Fig. 2, (Recall that the regular Bethe lattice $\{\infty, 4\}$ gives $R_{k=1} \rightarrow \infty$.) Now, we focus on the interval $0 \leq k \lesssim 20$, where $T_{\mathrm{f}}$ dominates. The bulk excess free energy increases locally around $T_{\mathrm{f}}$, which is not allowed for the free energy in Eq. (4). There is an inflection point, which is exactly positioned at $T=T_{\mathrm{t}}$. To investigate this feature in detail, we calculate the 'specific heat' for each $k(0 \leq k \lesssim 20)$ separately, as shown in Fig. 10.

Let us point out that the 'specific heat' for $k=1$ exhibits a maximum at the identical $T_{\mathrm{f}} \approx 0.339$, as plotted in the inset of Fig. 10. As $k$ consequently increases $(k=2,3,4, \ldots, \sim 20)$, there is no maximum anymore. However, the crossover in $\mathcal{C}_{\{\infty, 4\}}^{(2 \leq k \leq 20)}=0$ is a testament to the uniqueness of $T_{\mathrm{f}} \approx 0.339$ [notice the inflection point in $\mathcal{B}_{\{\infty, 4\}}^{(2 \leq k \leq 20)}$ which causes the crossover].

For $k \gtrsim 20$, the false phase transition is completely suppressed, and the abrupt decrease of $\mathcal{B}_{\{\infty, 4\}}^{(k \gtrsim 20)}$ accompanies the singularity, which converges to correct phase

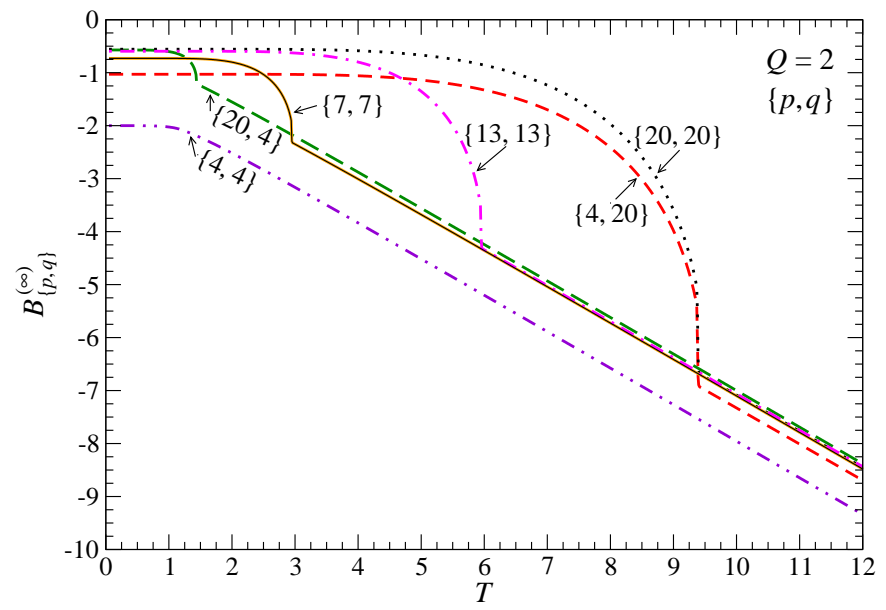

FIG. 11: (Color online) The bulk excess free energy of the Ising model on the selected set of the hyperbolic lattices $\{p, q\}$ when taking both the limits $L \rightarrow \infty$ and $k \rightarrow \infty$ for $h=0$. The bulk excess free energy remains unchanged for arbitrarily chosen $h_{b} \neq 0$.

transition in the bulk, $T_{\mathrm{pt}}^{(k \rightarrow \infty)}=1 / \ln 2$. We reached the complete numerical convergence of the bulk excess free energy after contracting $k \geq 2 \times 10^{3}$ layers.

\section{Ising model on hyperbolic $\{p, q\}$ lattices for $k \rightarrow \infty$}

An analogous dependence of the bulk excess free energy $\mathcal{B}_{\{p, q\}}^{(k)}$ on $k$ was confirmed numerically on different lattice geometries for the Ising model. Figure 11 depicts $\mathcal{B}_{\{p, q\}}^{(k)}$ for the Ising model on the several representative lattice geometries in the thermodynamic limit when $k \rightarrow \infty$. Imposing $h \neq 0$ on the Ising model on various hyperbolic $\{p, q\}$ shifts the bulk excess free energy as in Fig. 4 . The profile of the bulk excess free energy at $k \rightarrow \infty$ does not depend on $h_{b}$ when the Ising model is considered, provided that $h=0$ and $L \rightarrow \infty$. At sufficiently high temperatures, $T \gg T_{\mathrm{pt}}^{(\infty)}$, the slopes of $\mathcal{B}_{\{p, q\}}^{(k)}$ became identical for any $k \geq 0$. In other words, if we evaluate the normalized entropy per site in Eq. (5), then $S=1$ for $T \rightarrow \infty$. This is a numerical confirmation that the entropy of the $Q$-state spin models has to saturate at $\ln Q$ at high temperatures regardless of $k$ and the lattice geometry 15 .

\section{5-state Potts model on Bethe lattice for $k \rightarrow \infty$}

The $Q$-state Potts model with $Q>2$ exhibits a discontinuous (first-order) phase transition on hyperbolic lattices $\{p, q\}$. We have chosen the 5 -state Potts model on the Bethe lattice as a typical example. Discontinuous phase transitions characteristically exhibit a small temperature region, where two (or more) phases can co- 
exist. For instance, a simple order-disorder phase transition occurs for the $Q$-state Potts models, where the discontinuous phase transition $T_{\mathrm{pt}}$ can be located. In such a case, the free energy exhibits a kink at $T_{\mathrm{pt}}$ on the Euclidean lattices 32 . The phase transition is not critical since the correlation length does not diverge. The kink in the free energy means that taking its first derivative with respect to temperature results in a discontinuous function, a nonzero gap in the internal energy, at the phase transition. The gap $\Delta E\left(T_{\mathrm{pt}}\right)>0$ is proportional to the latent heat, which is a clear sign of the first-order phase transition. We do not study the $k$ dependence of the bulk excess free energy for the 5-state Potts model, as it is analogous to the 2-state Potts model. Instead, we focus on the special features of the first-order phase transition on the hyperbolic geometries.

If a model shows a discontinuous phase transition on the hyperbolic lattices, no kink in the free energy or the bulk excess free energy is found. The top graph in Fig. 12 shows the temperature dependence of the bulk excess free energy for the 5-state Potts model on the Bethe lattice when $L \rightarrow \infty$ and $k \rightarrow \infty$ at zero magnetic field $h$. We have considered a large variety of boundary conditions, which are determined by the boundary magnetic field $h_{b}$. We found out that the singular behavior of the bulk excess free energy for $k \rightarrow \infty$, yields many phase transitions $T_{\mathrm{pt}}\left(h_{b}\right)$, which are sensitive to the imposed magnetic field $h_{b}$. Within the interval $-\infty<h_{b}<+\infty$, we locate a phase-coexistence region on the finite-temperature interval $\Delta T$. The singular behavior in $\mathcal{B}_{\{\infty, 4\}}^{(\infty)}$ appears at $T_{\mathrm{pt}}$ and is accompanied by a discontinuous jump (denoted by the dotted line). The bottom graph zooms-in on the relevant part of the top graph, namely, the phase-coexistence region, and it shows, for which $h_{b}$ the phase transition $T_{\mathrm{pt}}\left(h_{b}\right)$ occurs. (We remark that there were no such discontinuities of the bulk excess free energy for the Ising model: the abrupt decrease with respect to $k$ was always continuous.)

Thus, the most emerging feature is the discontinuity of the bulk excess free energy while tuning the magnetic fields in the interval $10^{-3} \leq h_{b} \leq 0.7$. The finitetemperature interval determining the phase-coexistence region $\Delta T=T_{\text {high }}-T_{\text {low }}$ has a lower bound at $T_{\text {low }} \approx$ 0.7975 , which is stable for any boundary magnetic fields $h_{b} \lesssim 10^{-3}$ (including the negative fields), and an upper bound $T_{\text {high }} \approx 0.927$, which is saturated at $h_{b} \gtrsim 0.7$. Figure 13 extends our findings to more examples of the $Q$ state Potts models on various hyperbolic lattices. Since the Hausdorff dimension of the hyperbolic geometries $\{p, q\}$ is infinite for arbitrary $(p-2)(q-2)>4$, the first-order phase transition has to be realized for $Q \geq 3$. This is related to the fact that the $Q$-state Potts model on the three-dimensional cubic lattice 23136 exhibits firstorder phase transitions if $Q \geq 3$, whereas the Potts model on the two-dimensional Euclidean lattices shows this feature if $Q \geq 5$.

In general, the phase-coexistence region $\Delta T$ has a lower bound at $T_{\text {low }}\left(h_{b} \ll 0.1\right)$ and a finite upper bound
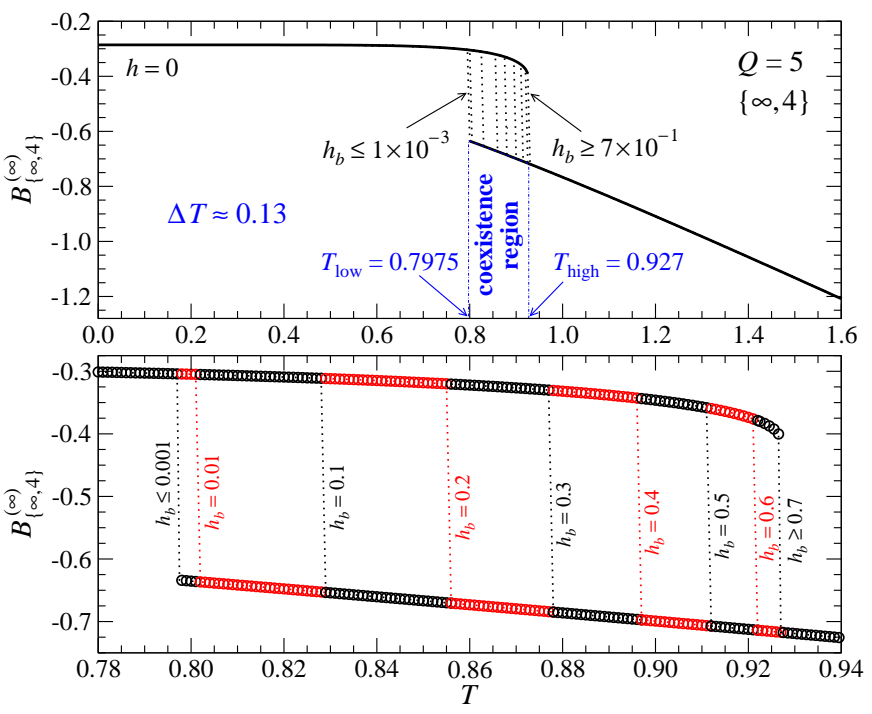

FIG. 12: (Color online) The temperature dependence of the bulk excess free energy for the 5-state Potts model on the Bethe lattice $\{\infty, 4\}$ after contracting the infinite number of the boundary layers $(k \rightarrow \infty)$. The phase transitions are shown as the discontinuities in $\mathcal{B}_{\{\infty, 4\}}^{(\infty)}$ for the particular boundary fields $h_{b}$. The discontinuities are marked by the dotted vertical lines. The graphs were constructed for $L \rightarrow \infty, k \rightarrow \infty$, and $h=0$.
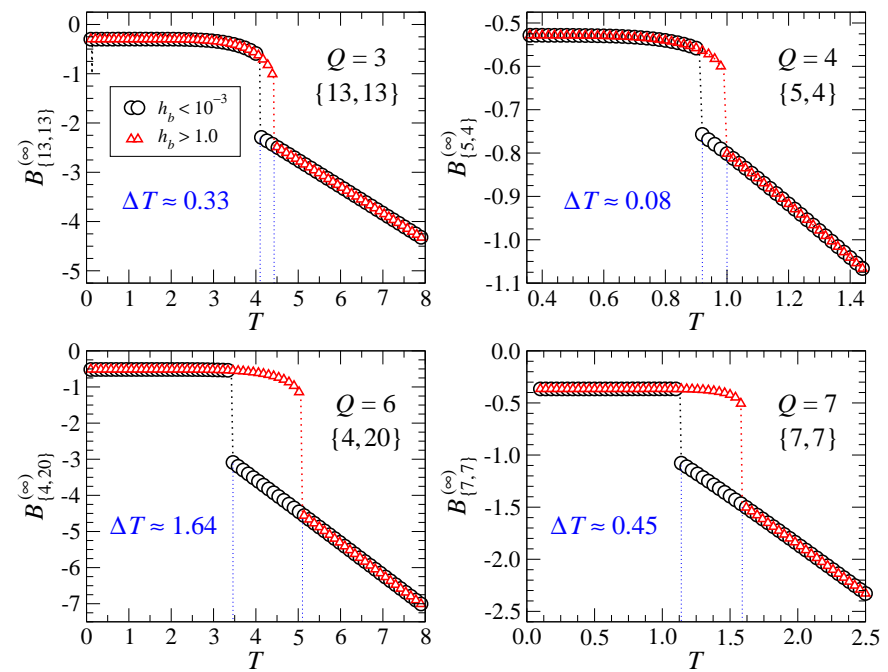

FIG. 13: (Color online) The temperature dependence of the bulk excess free energy for the $Q$-state Potts model for the selected Potts states $Q \geq 3$ and lattices $\{p, q\}$. The vertical dotted lines border the discontinuous phase coexistence regions $\Delta T$ for $L \rightarrow \infty, k \rightarrow \infty$, and $h=0$.

at $T_{\text {high }}\left(h_{b} \gg 0.1\right)$. The width of the phase coexistence region $\Delta T>0$ is primarily proportional to the lattice coordination number $q$ and the number of spin states $Q$, whereas there is a weak dependence on the $p$ (the larger the $q$ and/or $Q$, the wider $\Delta T$ ). We, therefore, conjecture that the first-order phase transition temperature $T_{\mathrm{pt}}$ is continuously adjustable within the interval 
$\Delta T$ by varying the boundary magnetic field $h_{b}$, which is achieved for the Potts models with $Q \geq 3$ and for an arbitrary hyperbolic geometry $\{p, q\}$.

\section{DISCUSSION AND REMARKS}

The reason for studying the complex behavior of the multistate spin models on the hyperbolic geometries lies in the potential to mimic the thermodynamic properties of various neural structures, social networks, anti de Sitter spaces, etc. We have introduced a way of specifying the novel function in the thermodynamic limit, the so-called bulk excess free energy per spin site, which is correlated with the number of contracted boundary spin layers of the entire lattice. At the same time, the bulk excess free energy remains sensitive to the magnetic field imposed on the outermost boundary layer. Recall that the phase transitions on the Euclidean lattices are not influenced by choosing any type of the boundary conditions in the thermodynamic limit. We have studied the boundary response to the bulk properties and the phase transitions in the classical multistate spin models on various hyperbolic lattices in the thermodynamic limit. We applied CTMRG to the $Q$-state Potts models and calculated the free energy per site with high accuracy. The Potts model has been selected for its availability to describe both second- and first-order phase transitions.

With respect to our results, there is no phase transition present on any hyperbolic lattice if the entire lattice in the thermodynamic limit is considered, provided that no boundary layers were contracted. To be more specific, there is a false phase transition induced by the huge boundary structure, which is strong enough to prevail over the correct phase transition observed deep inside the system if suppressing the boundaries. However, if a sufficient number of boundary layers are contracted (while keeping the infinite number of inner layers), the correct phase transition can appear in the bulk (known from the analytic solutions). Our results agrees with the only available analytic solution of spin models on Bethe lattices.

We have conjectured that the multistate spin systems on hyperbolic lattices, which exhibit a continuous (second-order) phase transition, exhibit a firm phasetransition point irrespective of the imposed boundary magnetic field $h_{b}$. Note that such spin models on hyperbolic lattice geometries belong to the mean-field universality class 1315 . If, however, a multistate spin system results in a discontinuous (first-order) phase transition in the bulk, the phase-transition point depends on the magnetic field $h_{b}$. In particular, if $h_{b}$ is varied on the outermost boundary layer, the phase-transition temperature $T_{\mathrm{pt}}\left(h_{b}\right)$ changes continuously within the tempera- ture region $\Delta T>0$ for any $Q \geq 3$ Potts models on the hyperbolic geometries $\{p, q\}$.

With respect to our recent studies of quantum Ising and Heisenberg models on hyperbolic lattices ${ }^{30 \mid 31}$, which exhibit analogous physical properties to those of the classical spins model, we expect the presence of identical features. To prove this conjecture, we intend to perform extensive numerical studies on quantum systems. The multistate Potts models on non-Euclidean lattices were studied in this paper for their capability to mimic realistic interacting systems, such as neural systems, social Internet networks, etc., in which the boundary influences the behavior in the central part. The current remarkable physical feature opens leads to an alternative view of the importance of boundary effects, as we have shown that one can continuously control the thermodynamic properties of certain types of complex systems on hyperbolic geometries.

A condensed-matter view point on the AdS-CFT correspondence is known to restrict a negatively curved space geometry (AdS) to a preferred coordinate system, i.e., a lattice 37 . We reduced the problem to an infinite set of two-dimensional curved hyperbolic surfaces, where the underlying lattice geometry $\{p, q\}$ varies by changing the two integer lattice parameters $p$ and $q$. The impact of the $\{p, q\}$ geometry on the phase transitions with respect to the boundary effects has been studied. Making use of the free-energy language, the boundary structure of the hyperbolic geometry is naturally incorporated into the solution to carry the essential feature of AdS space. Our intention for future studies is to calculate the von Neumann entanglement entropy of a subsystem, e.g. a bulk, for the quantum Heisenberg and transverse field Ising models on $\{4, q>3\}$ lattice geometries 38 . The impetus for this calculation lies in the concept of holographic entanglement entropy ${ }^{9}$, in which a nongravitational theory is expected to exist on the boundary of the (bulk) subsystem of $(d+1)$-dimensional hyperbolic spaces. The entanglement entropy, which is related to a reduced density matrix of the bulk subsystem, provides an appropriate measure of the amount of information within the AdS-CFT correspondence. Hence, the entropy is proportional to the boundary (minimal area surface) within AdS space, which links the duality with the corresponding $d$-dimensional bulk region defined in CFT.

\section{Acknowledgments}

This work was supported by Vedecká Grantová Agentúra MŠVVaŠ SR a SAV (VEGA-2/0130/15) and Agentúra na Podporu Výskumu a Vývoja (QIMABOSAPVV-0808-12).

\footnotetext{
* Electronic address: andrej.gendiar@savba.sk
} 
K. Agawa, Appl. Phys. Lett. 85, 5287 (2004).

2 F. Liang, L. Guo, Q. P. Zhong, X. G. Wen, C. P. Chen, N. N. Zhang, and W. G. Chu, Appl. Phys. Lett. 89, 103105 (2006).

3 A. Cabot, A. P. Alivisatos, V. F. Puntes, L. Balcells, O. Iglesias, and A. Labarta, Phys. Rev. B 79, 094419 (2009).

4 W. A. Moura-Melo, A. R. Pereira, L. A. S. Mol, and A. S. T. Pires, Phys. Lett. A 360, 472 (2007).

${ }^{5}$ V. A. Kazakov, Phys. Lett. A 119, 140 (1986).

${ }^{6}$ C. Holm and W. Janke, Phys. Lett. B 375, 69 (1996).

7 D. Krioukov, F. Papadopoulos, A. Vahdat, and M. Boguñà, Phys. Rev. E 80, 035101 (2009).

8 D. Krioukov, F. Papadopoulos, M. Kitsak, A. Vahdat, and M. Boguñà, Phys. Rev. E 82, 036106 (2010).

${ }^{9}$ G. 't Hooft, in World Scientific Series in 20th Century Physics, Vol. 4, edited by A. Ali, J. Ellis, and S. RandjbarDaemi (World Scientific, 1993).

10 L. Susskind, J. Math. Phys. (N.Y.) 36, 6377 (1995).

11 S. Ryu and. T. Takayanagi, Phys. Rev. Lett. 96, 181602 (2006).

12 J. Maldacena, Adv. Theor. Math. Phys. 2, 231 (1998); Int. J. Theor. Phys. 38, 1113 (1999).

13 K. Ueda, R. Krcmar, A. Gendiar, and T. Nishino, J. Phys. Soc. Jpn. 76, 084004 (2007).

14 T. Nishino and K. Okunishi, J. Phys. Soc. Jpn. 65, 891 (1996); T. Nishino and K. Okunishi, J. Phys. Soc. Jpn. 66, 3040 (1997).

15 M. Serina, J. Genzor, Y. Lee, and A. Gendiar, Phys. Rev. E 93, 042123 (2016).

${ }^{16}$ H. Shima and Y. Sakaniwa, J. Stat. Mech., P08017 (2006).

17 H. Shima and Y. Sakaniwa, J. Phys. A: Math. Gen. 39, 4921 (2006).

18 S. K. Baek, P. Minnhagen, and B. J. Kim, EPL 79, 26002 (2007)

19 Y. Sakaniwa and H. Shima, Phys. Rev. E 80, 021103
(2009)

${ }^{20}$ I. Hasegawa, Y. Sakaniwa and H. Shima: Surf. Sci. 601, 5232 (2007).

21 R. Mosseri and J. F. Sadoc, J. Physique - Lettres 43, L249 (1982).

22 J. W. Anderson, Hyperbolic Geometry (2nd edition, Springer, London, 2005).

${ }^{23}$ F. W. Wu, Rev. Mod. Phys. 54 (1982) 235.

24 S. R. White, Phys. Rev. Lett. 69, 2863 (1992).

25 U. Schollwöck, Rev. Mod. Phys. 77, 259 (2005).

26 R. Krcmar, A. Gendiar, K. Ueda, and T. Nishino, J. Phys. A 41, 125001 (2008).

27 R. J. Baxter, Exactly Solved Models in Statistical Mechanics (Academic Press, London, 1982.)

28 A. Gendiar, R. Krcmar, S. Andergassen, M. Daniška, and T. Nishino, Phys. Rev. E 86, 021105 (2012).

29 A. Gendiar, M. Daniška, R. Krcmar, and T. Nishino, Phys. Rev. E 90, 012122 (2014).

30 M. Daniška and A. Gendiar, J. Phys. A: Math. Theor. 48, 435002 (2015).

31 M. Daniška and A. Gendiar, J. Phys. A: Math. Theor. 49, 145003 (2016).

32 J. Genzor, V. Bužek, and A. Gendiar, Physica A 420, 200 (2015).

${ }^{33}$ F. Verstraete and J. I. Cirac, Phys. Rev. B 73, 094423 (2006).

34 C. A. Schwerdtfeger and D. A. Mazziotti, J. Chem. Phys. 130, 224102 (2009).

35 V. Zauner, D. Draxler, L. Vanderstraeten, J. Haegeman, and F. Verstraete, arXiv:1412.7642.

36 A. Gendiar and T. Nishino, Phys. Rev. E 65, 046702 (2002)

37 P. W. Anderson, Phys. Today 66(4), 9 (2013).

38 M. Daniška and A. Gendiar (unpublished). 